\title{
Kinematic Characteristics of Postural Control during Reaching in Preterm Children with Cerebral Palsy
}

\author{
JOLANDA C. VAN DER HEIDE, JOHANNA M. FOCK, BERT OTTEN, ELISABETH STREMMELAAR, \\ AND MIJNA HADDERS-ALGRA
}

Department of Neurology [J.C.v.d.H., J.M.F., M.H.-A.], Department of Human Movement Sciences [B.O], Department of Pediatrics [E.S.], University Hospital Groningen, 9713 GZ Groningen, The Netherlands

\begin{abstract}
ABST
The relationships between kinematic characteristics of sitting
posture during reaching movements of the dominant arm and 1)
the kinematics of the reaching movement itself and 2) functional
performance during daily life activities (PEDI) were assessed in
51 sitting preterm children with cerebral palsy (CP). The children
were 2-11 y, 33 had spastic hemiplegia (SH) and 18 bilateral CP
(Bi-CP). The data were compared with those of 26 typically
developing children (TD). Sitting posture before the onset of
reaching of children with CP differed from that of TD children:
they sat with a more reclined pelvis and a more collapsed trunk.
The more reclined pelvic position was associated with a better
quality of reaching movements. The different sitting postures of
pelvis and trunk were not related to functional performance
during daily life activities. Displacement of the head, trunk, and
pelvis of the children with CP did not differ from that of the TD
children. Nevertheless, in the children with CP a more stable
head, a more mobile trunk, and a more stable pelvis were related
\end{abstract}
Postural control is a prerequisite to perform daily activities such as walking and reaching. It is also assumed that the quality of reaching depends on the adequacy of postural control $(1,2)$. The link between reaching and postural control has a firm neurophysiological basis as the control of both types of behavior is mediated primarily by the medially descending brain stem systems (3). The tight link is reflected by the presence of postural adjustments accompanying reaching movements (4). In addition, it has been shown that the emergence of reaching movements is related to postural competence $(5,6)$. But, quite surprisingly, the relationship between the quality of reaching and adequacy of postural control seldom has been a subject of research. The studies of Fallang et al. (7-9), which addressed

Received March 17, 2004; accepted January 13, 2005.

Correspondence: Mijna Hadders-Algra, M.D., Ph.D., University Hospital Groningen, Developmental Neurology, Hanzeplein 1, 9713 GZ Groningen, The Netherlands; e-mail: m.hadders-algra@med.rug.nl

In this article, we use the term dominant arm to refer to the arm tested, i.e. the least involved arm in children with CP and the dominant arm in TD children.

DOI: $10.1203 / 01 . p d r .0000176834 .47305 .26$ to better functional performance and/or a better quality of reaching. This suggests that physiotherapeutic guidance of children with $\mathrm{CP}$ should focus rather on the latter postural parameters than on the different sitting posture of pelvis and trunk. (Pediatr Res 58: 586-593, 2005)

Bbbreviations
CP, cerebral palsy
EMG, electromyography
GMFCS, Gross Motor Function Classification System
MU, movement unit
PEDI, Pediatric Evaluation of Disability Inventory
PVL, periventricular leukomalacia
SH, spastic hemiplegia
TD, typically developing

the relationship between reaching and postural control in supine position in full-term and preterm infants, are exceptions to this rule. The studies indicated that in infants aged 4 and 6 mo. a better postural control is related to a better reaching performance. Hopkins and Rönnqvist (10), who studied typically developing infants, demonstrated that providing sitting infants aged 6 mo with extra support at the pelvic girdle, resulted in reaching movements with less movement units. Movement units (MU) are submovements of the reaching movement and are determined with the help of peaks in the velocity profile of the reaching hand $(11,12)$.

From a previous study, we know that children with $\mathrm{CP}$ are hampered by dysfunctional postural control during reaching (13). Children with CP especially have problems in adapting the degree of contraction of the postural muscles to the characteristics of the situation. For instance, a substantial number of children with $\mathrm{CP}$, in particular children with a bilateral spastic form of $\mathrm{CP}$, cannot modulate muscle contraction (as registered on EMG) during reaching to kinematic information on initial body configuration, i.e. the sensory information on 
sitting position. Thus, we wondered whether postural dysfunction in children with $\mathrm{CP}$ would be related to a worse quality of reaching.

Therefore, the aim of our study was to see whether in children with $\mathrm{CP}$ kinematics of postural control are related to kinematic quality of reaching. To this end, we studied 58 children with spastic $\mathrm{CP}$ aged $2-11$ y with $\mathrm{SH}$ or a Bi-CP and 29 age-matched TD children. Kinematics of the arm, head, trunk, and pelvis were recorded while the children made reaching movements with their dominant (in TD children) or least involved (in children with CP) arm (1) in a sitting position. In addition to the kinematic postural data, we had information on the child's ability to modulate postural EMGactivity with respect to initial body configuration, i.e. the position of the head, trunk, and pelvis at the beginning of the arm movement (see 13). We first addressed the question whether children with $\mathrm{CP}$ and typically developing children differ in the kinematics of postural control (initial sitting position and postural displacement) and, if so, whether the kinematics of postural control of children with $\mathrm{CP}$ are influenced by a) type of $\mathrm{CP}(\mathrm{SH}$ versus $\mathrm{Bi}-\mathrm{CP}), \mathrm{b})$ age, c) gestational age at birth, d) severity and uni- or bilateral nature of the lesion on the neonatal ultrasound scan of the brain, e) the degree of spasticity of the reaching arm as measured by the modified Ashworth scale (14), f) clinical severity of the disability. Next, we investigated whether postural performance in children with $\mathrm{CP}$ is related to functional performance in daily life as measured by the PEDI (15). Finally, we evaluated whether the parameters of postural control, such as kinematic variables on initial body configuration and body displacement and the EMG-parameters on the capacity to modulate postural muscle activity, are related to the parameters of reaching. We also addressed the questions 1) whether the possible relationships between postural control and reaching differ for the groups of children with $\mathrm{SH}$ or Bi-CP and the TD children, and 2) whether these relationships in the children with $\mathrm{CP}$ are affected by age, severity and uni- or bilateral nature of the lesion on the neonatal ultrasound scan of the brain, gestational age at birth, degree of spasticity and severity of disability.

\section{METHODS}

Participants. The study group consisted of 58 children with CP, aged 2-11 y. Thirty-four had a spastic hemiplegia-17 a right-sided one and 17 a left-sided one-and 24 had a bilateral form of spastic CP. Spasticity was associated with dyskinesia in seven children and with ataxia in five. Children with a severe visual impairment and those who were not able to reach out for an object were excluded from the study. All but one of the children with SH were able to walk without assistive devices; they had a level I $(n=33)$ or a level IV $(n=1)$ disability according to the GMFCS (16). In the group of children with Bi-CP, 9 children could walk without assistive devices, 10 could walk with the help of assistive mobility devices (GMFCS level III), and 5 children had limited self-mobility (GMFCS level IV-V).

All children with $\mathrm{CP}$ were born preterm and admitted to the neonatal intensive care unit of the Groningen University Hospital. Gestational age and weight at birth did not differ for the groups of children with SH and those with $\mathrm{Bi}-\mathrm{CP}$. In the majority of children, serial neonatal brain ultrasound scans had been made with a high-resolution $7.5 \mathrm{MHz}$ transducer until 1 mo post-term. After this age, a 5-MHz transducer was used. The children were scanned during the first weeks after birth at various intervals (ranging from $3 \mathrm{~d}$ to $2 \mathrm{wk}$ ). Periventricular hemorrhages and PVL in the preterm children were classified according the methods of Levene et al. (17) and De Vries et al. (18), respectively. The severity of the brain lesions was classified as no lesion, as mild in case of the presence of a grade 1 or $2 \mathrm{PVL}$ and/or hemorrhage grade 1 or 2 or as severe in case of a PVL grade 3 and/or hemorrhage grade 4 . Another variable addressed the unilateral or bilateral nature of the brain lesion.

The reference group consisted of 29 children with a typical motor development born at term. Nine children of the TD group were 2-4 y, 10 children were 5-7 y, and another 10 were 8-11 y (see 19). TD children aged at least $4 \mathrm{y}$ were assessed with the Movement ABC (20). They all scored above the 15th percentile, which indicates that the children showed age-adequate motor behavior. All parents gave informed consent. The Medical Ethics Committee of the University Hospital Groningen approved the study.

Procedure. The majority of children sat on a table without back and foot support. Some children needed extra support to be able to carry out the test. Back and foot support was provided in 5 of the 12 children with severe CP. Foot support only was given to three children with severe $\mathrm{CP}$ and one young child with moderately severe $\mathrm{CP}$. The examiner presented an attractive small object in the midline at arm's length distance of the subject. The instruction was to grasp the object with the dominant hand at a natural self-paced speed. We deliberately chose the dominant arm, as we were interested in postural control during daily life activities. The dominant hand was defined as the hand with which the child preferred to write or draw. Ten to 20 trials were performed. Before testing, the children carried out some exercise trials.

Movements were recorded kinematically with an ELITE opto-electronic movement recording system (BTS, Milan, Italy) in a four-camera configuration at a sampling frequency of $50 \mathrm{~Hz}$. Reflective markers were placed at the side of the body of the dominant hand on the following landmarks: 1) condyle of mandible; 2) $1 \mathrm{~cm}$ in front of the angle of mandible; spinous processes at 3) C7, 4) T10, and 5) L5; 6) anterior superior iliac spine; 7) proximal edge of the greater trochanter; 8) acromion; 9) epicondyle of the radius; and 10) styloid process of the radius (Fig. 1). Sampling of the kinematic data started some seconds before toy presentation and lasted for 8-12 s, depending on the reaching velocity of the child. As kinematic recording in young children often goes hand in hand with a considerable loss of data, we included children only into the analyses when at least three appropriate kinematic trials could be achieved (Table 1). Data of seven children with CP [one child with SH with GMFCS level I, and six children with Bi-CP (GMFCS level I: $n=1$, level II: $n=1$, level III: $n=2$, level IV: $n=2$ )] and three TD children did not meet the three trials criterion (two belonging to the youngest age group and one child of $5 \mathrm{y}$ old).

Furthermore, simultaneously multiple surface EMG-recordings were made of the postural muscles. The results of the EMG data have been reported elsewhere (13). On the basis of the EMG results, the children were classified

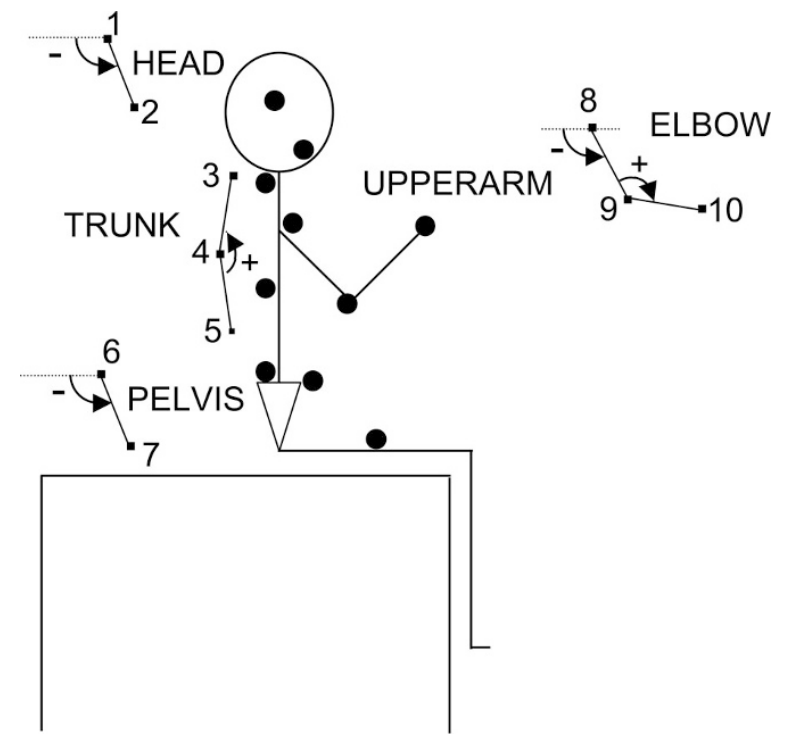

Figure 1. Schematic representation of the testing condition. The dots denote the position of the ELITE markers and the inserts indicate how the angles of the head, trunk, pelvis, upper arm, and elbow in the sagittal plane were calculated. Head: more negative values indicate more retroflexion of the head. Trunk: more positive values indicate straightening of the back. Pelvis: more negative values indicate retroflexion. Upper arm: more negative values indicate more shoulder anteflexion. Elbow: more positive values indicate more arm extension. 
Table 1. Number of trials per individual with successful kinematic recording

\begin{tabular}{clrcc}
\hline & & & \multicolumn{2}{c}{ Number of trials } \\
\cline { 3 - 5 } Age & \multicolumn{1}{c}{ Group } & No. & Median & Range \\
\hline \multirow{2}{*}{$2-4$ y } & Children with SH & 8 & 17 & $4-10$ \\
& Children with Bi-CP & 4 & 16 & $4-9$ \\
& TD & 7 & 14 & $3-9$ \\
$5-7$ y & Children with SH & 12 & 15 & $3-9$ \\
& Children with Bi-CP & 7 & 14 & $3-10$ \\
& TD $8-11$ y & 9 & 19 & $5-11$ \\
& Children with SH & 13 & 16 & $3-10$ \\
& Children with Bi-CP & 7 & 16 & $5-11$ \\
& TD & 10 & 11 & $4-13$ \\
\hline
\end{tabular}

as having or not having the ability to modulate EMG-amplitude of the dorsal postural muscles (neck and trunk extensor muscles) with respect to body configuration at reaching onset (13).

The whole session was recorded on video. After the reaching sessions, neurologic condition, functional performance, and degree of spasticity of the children were evaluated. On the basis of the neurologic examination, three classes of severity were distinguished: mild ( $\mathrm{SH}, n=24$; Bi-CP, $n=6$ ), moderate ( $\mathrm{SH}, n=8$; Bi-CP, $n=5$ ), and severe ( $\mathrm{SH}, n=1$; Bi-CP, $n=7$ ), indicating that posture, motility, muscle tone, and reflexes were affected to a limited, moderate, and severe extent, respectively. Functional performance in self-care, transfers, and social abilities was assessed during a telephone interview some days after the postural evaluation by means of the standardized Dutch PEDI (15). Degree of spasticity of the biceps brachii muscle of the dominant arm was assessed with the help of the modified Ashworth scale (14)

Data analysis. The video recordings were used to select reaching movements with the dominant arm, during which the child was in an appropriate state of attention. Such a selection procedure was needed in particular in the youngest children.

The onset of the reaching movements was determined on the basis of the kinematic data. Off-line kinematic analysis was carried out with the help of the software package DataMonster 2.0 (E. Otten, Department Human Movement Sciences, University of Groningen) (21). Kinematic analysis consisted of the calculation of spatial angles for the head (by a vector between markers 1 and 2), the pelvis (by a vector between markers 6 and 7), and the upper arm (by a vector between markers 8 and 9) in relation to the horizontal plane. In addition, the trunk angle was defined by calculating the angle of the two intersecting vectors between markers 3 and 4 and markers 4 and 5, and the elbow angle as the angle of the two vectors between markers 8 and 9 and markers 9 and 10 (Fig. 1). Arm movement onset was defined as the moment at which the velocity of the wrist increased more than $5 \%$ of peak velocity, while the moment at which wrist velocity declined to $5 \%$ of peak velocity at the farthest distance in space relative to the beginning of the movement was considered as the end of the movement. In the kinematic analysis, only trials with a clearly demarcated start and stop were included (Table 1). The analysis of the kinematics of postural control focused on 1) angular positions at movement onset and 2) angular displacements during the entire duration of the reaching movement. For the analysis of the kinematics of reaching we focused on a) reaching duration, b) maximum reaching velocity, as revealed by the $\mathrm{V}_{\max }$ of the wrist, c) index of curvature of the reaching movement, as indicated by the ratio of the actual length of the reaching path and the length of the straight line between starting and stopping position (expressed as a percentage) (22), d) the proportion of trials during which the reaching movement consisted of one MU, and e) the length of the first MU (the transport MU) relative to total movement path (expressed as a percentage). The MU were distinguished with the help of the wrist velocity peak profile. An MU consisted of one acceleration and one deceleration in the velocity profile of the wrist marker $(11,12)$.

Statistics. Statistical analyses were performed using the computer package SPSS (version 10.1, SPSS Inc., Chicago, IL). The majority of analyses were performed on child-level, meaning that for the kinematic variables first the child's mean value was calculated on the basis of which further analyses were performed. For the evaluation of the effect of type of $\mathrm{CP}$ (SH or Bi-CP), effect of age and the presence of additional back or foot support the nonparametric Mann-Whitney test was used. To establish the influence of the uni- or bilateral nature of the brain lesion on the ultrasound scan on the kinematic parameters of postural control, the Kruskal-Wallis test was used. For the relation between clinical characteristics, such as the PEDI and the severity of brain lesion, and the kinematic parameters and for the relation between the kinematics of posture and those of reaching, Spearman's rho was calculated. To be able to differentiate between the effects of gestational age (born before $28 \mathrm{wk}$ or $\geq 28$ wk) (13) versus severity of brain lesion on the kinematic parameters of postural control a multiple regression analysis was performed.

To be able to evaluate the effect of age while taking into account the effect of group and the severity of disability, ANOVA was used. Similarly, to evaluate the effect of the ability to modulate postural muscle activity on the basis of information on initial body configuration while taking into account the effect of age and group, ANOVA was again applied. We realize that the ANOVA is a parametric test and thus nonoptimal for the present set of data, but it was the only way to get some idea on the effect of multiple factors on the kinematic parameters.

Throughout the analyses, we considered in the nonparametric tests differences with $p$ values $<0.05$ and in the parametric tests differences with $p$ values $<0.01$ as statistically significant.

\section{RESULTS}

Kinematics of postural control. Before addressing the differences in the kinematics of postural control between the TD children and the children with $\mathrm{CP}$, we first describe the effect of age on postural parameters in the TD children. In the TD children, age only affected the degree of straightness of the trunk and the stability of the head during reaching. The youngest TD children had a straighter position of the trunk compared with the $5-11$ y olds (median values: $2-4$ y olds, $159^{\circ} ; 5-7$ y olds, $152^{\circ} ; 8-11$ y olds, $142^{\circ}$; respectively $p<0.05, p<0.01$ ) and the youngest TD children showed less stability of the head during reaching compared with the 8-11 y olds (displacement of the head, median values: $2-4 \mathrm{y}$ olds, $9.5^{\circ}$ versus $8-11 \mathrm{y}$ olds, $3.4^{\circ}, p<0.05 ; 5-7$ y olds, $3.8^{\circ}(\mathrm{NS})$.

Initial body configuration differed between the three groups (Fig. 2). The children with $\mathrm{CP}$ sat with a more flexed trunk (median values: TD, 153 $; \mathrm{SH}, 145^{\circ}$; Bi-CP, $142^{\circ} ; p<0.05$ ) and had their pelvis, in particular the children with $\mathrm{SH}$, in a more reclined position (median values: $\mathrm{TD},-108^{\circ} ; \mathrm{SH},-115^{\circ}$; $\left.\mathrm{Bi}-\mathrm{CP},-111^{\circ} ; p<0.05\right)$ than the TD children. The children with Bi-CP had a more flexed elbow position than the children with $\mathrm{SH}$ and the TD children (especially in the 5-7 y olds; median values: $\mathrm{TD}, 150^{\circ}$; $\mathrm{SH}, 134^{\circ}$; $\mathrm{Bi}-\mathrm{CP}, 122^{\circ} ; p<0.05$ ). Furthermore, the children with Bi-CP had a more retroflexed position of the shoulder than the TD children (especially the $2-4$ y olds; median values: TD, $-106^{\circ}$; Bi-CP, $-97^{\circ} ; p<0.05$ ). In general, the angular displacements during the reaching movement did not differ between the three groups. The only difference found was that children with $\mathrm{SH}$ displaced their upper arm more during reaching than TD children (median values: $51.7^{\circ}$ versus $40.6^{\circ}, p<0.01$ ). In addition, children with SH tended to show a larger displacement of the head during reaching than the TD children (median values: $6.9^{\circ}$ versus $4.4^{\circ}, p=0.08$ ), but this difference failed to reach statistical significance.

The sitting position at the onset of reaching was related to the severity of disability. Children with severe CP held their head more in anteflexion than the children with a mild or moderate form of $\mathrm{CP}$ (mean values: severe, $-95^{\circ}$; moderate, $-109^{\circ}$; mild $\left.-101^{\circ} ; \mathrm{F}_{2,8}=10.3, p<0.01\right)$. This was in particular true for the $2-4$ y olds and the $8-11$ y olds (age group * severity $\left.\mathrm{F}_{4,8}=6.3, p<0.01\right)$. Children with severe $\mathrm{CP}$ also sat with a more flexed trunk (mean values: severe, $137^{\circ}$; moderate, $143^{\circ}$; mild, $146^{\circ} ; \mathrm{F}_{2,8}=18.2, p<0.01$ ). Children with moderate form of $\mathrm{CP}$ had a less reclined pelvis position 


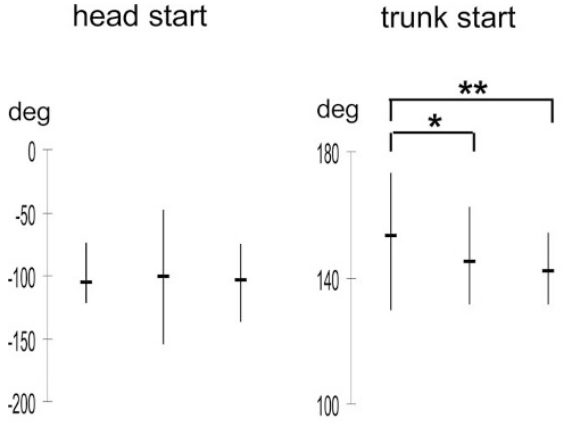

TD SH Bi-CP pelvis start

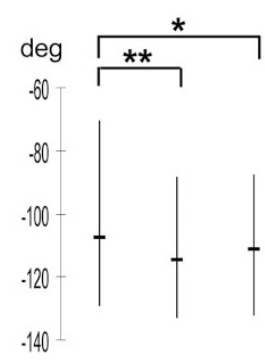

upper-arm start

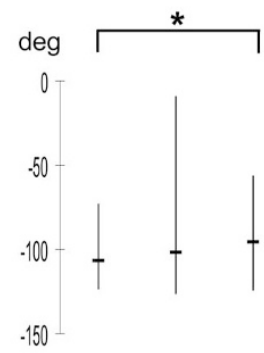

elbow start

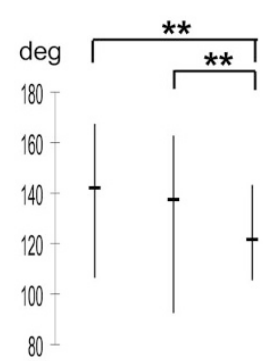

TD SH Bi-CP

Figure 2. Kinematic characteristics of postural control in children with and without CP. Medians (horizontal bars) and ranges (vertical bars) of the kinematic parameters of the initial body position. Mann-Whitney, ${ }^{*} p<0.05,{ }^{* *} p<0.01$. More negative values for head start, pelvis start, and upper arm start indicate an increase in retroflexion for head and pelvis and an increase in anteflexion of the shoulder for the upper arm. An increase in the values of trunk and elbow reflect an increase in extension.

(mean values: severe, $-115^{\circ} \mathrm{p}$; moderate, $-110^{\circ}$; mild, $-116^{\circ}$; $\left.\mathrm{F}_{2,8}=11.6, p<0.01\right)$. The latter difference was especially clear in the 5-11 y olds (age group $*$ severity $\mathrm{F}_{4,8}=9.4, p<$ 0.01). In addition, children with severe $\mathrm{CP}$ started the reaching movement with the upper arm held in a more retroflexed position than the children with a mild or moderate form of $\mathrm{CP}$ (mean values: severe, $-83^{\circ}$; moderate, $-97^{\circ}$; mild, $-100^{\circ} ; \mathrm{F}_{2,8}$ $=7.2, p<0.01)$. In particular, the $5-11 \mathrm{y}$ olds held their upper arm in a retroflexed position (age group $*$ severity $\mathrm{F}_{4,8}=5.2$, $p<0.01$ ). Furthermore, the children with severe $\mathrm{CP}$ held their elbow in a more flexed position (mean values: severe, $122^{\circ}$; moderate, $124^{\circ}$; mild, $136^{\circ} ; \mathrm{F}_{2,8}=16.6, p<0.01$ ). Especially the 5-7 y olds with severe CP showed a more flexed elbow position (age group * severity $\mathrm{F}_{4,8}=4.5, p<0.01$ ). Little relation was found between the severity of disability and body displacement during reaching. Only for the angular displacement of the pelvis was a main effect for severity (mean values: severe, $5.5^{\circ}$; moderate, $3.0^{\circ}$; mild, $4.3^{\circ} ; \mathrm{F}_{2,8}=6.9, p<0.01$ ) and an interaction effect found between the age group and the severity of disability (age group $*$ severity $\mathrm{F}_{4,8}=10.2, p<$ 0.01 ). The latter result meant that children with moderate $\mathrm{CP}$ in particular at the age of 2-4 y had a smaller angular displacement of the pelvis than children with either mild or severe CP. In addition, severity of disability tended to affect the stability of the head during reaching (mean values of head displacement: severe, $9.8^{\circ}$; moderate, $5.9^{\circ}$; mild, $7.8^{\circ} ; \mathrm{F}_{2,8}=4.7, p=0.01$ ). The kinematics of postural control of children who received additional postural support during testing was similar to that of the children with severe $\mathrm{CP}$ who did not receive extra support. Only the children with severe or moderate $\mathrm{CP}$ who received additional support tended to have less displacement of the pelvis than children with severe $\mathrm{CP}$ who were tested without extra support (median values: $1.2^{\circ}$ versus $6.1^{\circ}, p=0.08$ ).

Neonatal characteristics and kinematics of postural control. Kinematics of postural control in children with $\mathrm{CP}$ was related to some extent to the severity of the lesion on the neonatal ultrasound scan of the brain. Little relation was found between severity of brain lesion and initial body position. Only in the subgroup of children with $\mathrm{SH}$ was a relation found between a more severe brain lesion and a higher degree of retroflexion of the upper arm (rho $=0.37, p<0.05)$. The severity of brain lesion was related to the stability of the head during reaching. A more severe brain lesion was associated with more displacement of the head (rho $=0.35, p<0.05$ ). This was also true for the subgroup of children with $\mathrm{SH}$ (rho = 0.44, $p<0.05$ ), but not for the subgroup of children with $\mathrm{Bi}-\mathrm{CP}($ rho $=0.18, \mathrm{NS})$. The relationships between the presence of a brain lesion and the kinematics of posture were not affected by the unilateral or bilateral nature of the lesion.

In the children with $\mathrm{CP}$, gestational age at birth was not related to the kinematics of postural control. When taking into account both gestational age at birth and the severity of the brain lesion on the ultrasound scan by using a multiple regression analysis, the position of the upper arm at the start of the reaching movement and the displacement of the head during the reaching movement were related to the severity of the brain lesion $(p<0.05)$. Both results were brought about by the children with SH. In the multivariate statistics, gestational age at birth was not related to any of the kinematic variables of postural control.

Functional measurements and kinematics of postural control. In the children with CP, initial position of the trunk and pelvis were not clearly related to scores on the PEDI (Table 2). In the children with $\mathrm{SH}$, a less anteflexed initial position of the head was related to better PEDI scores. A similar relationship was absent in the children with Bi-CP. In this group, a more anteflexed position of the head tended to be related to better PEDI scores. Initial position of the upper arm and elbow were related to some extent to PEDI scores. In particular, in children with $\mathrm{SH}$, a more retroflexed starting position of the upper arm was related to worse PEDI scores. Displacement of the head, trunk, and upper arm was not or minimally related to PEDI scores. However, less displacement of the pelvis and less displacement of the elbow were related to better PEDI scores. This was especially true for children with $\mathrm{SH}$.

Kinematics of postural control in children with $\mathrm{CP}$ was related to some extent to the degree of spasticity of the reaching arm measured by the modified Ashworth scale. A higher degree of spasticity was related to a more flexed initial position of the elbow (rho $=-0.32, p<0.05$ ), but not to the degree of angular displacement of the shoulder and elbow. 
Table 2. Spearman rank correlations between the subscales and total scores of the PEDI and the kinematic parameters for the children with $C P$

\begin{tabular}{|c|c|c|c|c|c|c|c|}
\hline & \multicolumn{7}{|c|}{ All children with $\mathrm{CP}$} \\
\hline & $\begin{array}{l}\text { PEDI } \\
\text { fssc }\end{array}$ & $\begin{array}{l}\text { PEDI } \\
\text { fsmob }\end{array}$ & $\begin{array}{l}\text { PEDI } \\
\text { fssoc }\end{array}$ & $\begin{array}{l}\text { PEDI } \\
\text { casc }\end{array}$ & $\begin{array}{l}\text { PEDI } \\
\text { camob }\end{array}$ & $\begin{array}{l}\text { PEDI } \\
\text { casoc }\end{array}$ & $\begin{array}{l}\text { PEDI } \\
\text { total score }\end{array}$ \\
\hline Head start & -0.11 & -0.14 & -0.22 & -0.21 & -0.02 & -0.16 & -0.16 \\
\hline Trunk start & 0.13 & 0.23 & -0.15 & -0.11 & 0.30 & -0.17 & 0.10 \\
\hline Pelvis start & 0.05 & 0.07 & 0.02 & -0.04 & 0.16 & 0.17 & 0.08 \\
\hline Upper arm start & -0.37 & -0.41 & -0.36 & -0.35 & -0.35 & -0.22 & -0.39 \\
\hline Elbow start & -0.29 & -0.39 & 0.05 & 0.19 & 0.22 & 0.01 & 0.27 \\
\hline Delta head & -0.11 & 0.15 & -0.06 & -0.16 & -0.02 & -0.06 & -0.14 \\
\hline Delta trunk & 0.06 & 0.01 & 0.01 & -0.07 & 0.13 & 0.03 & 0.03 \\
\hline Delta pelvis & -0.34 & -0.36 & -0.44 & -0.37 & 0.02 & -0.12 & -0.37 \\
\hline Delta upper arm & 0.01 & -0.03 & -0.05 & -0.00 & -0.06 & -0.05 & 0.00 \\
\hline Delta elbow & -0.40 & -0.37 & -0.35 & -0.36 & -0.09 & -0.33 & -0.38 \\
\hline
\end{tabular}

Children with $\mathrm{SH}$

PEDI PEDI PEDI PEDI PEDI PEDI PEDI

fssc fsmob fssoc casc camob casoc total score

\begin{tabular}{lrrrrrrr}
\hline Head start & -0.30 & $\mathbf{- 0 . 3 5}$ & $\mathbf{- 0 . 3 7}$ & $\mathbf{- 0 . 3 9}$ & -0.14 & $\mathbf{- 0 . 4 7}$ & $\mathbf{- 0 . 3 5}$ \\
Trunk start & 0.09 & 0.10 & -0.06 & -0.20 & 0.23 & -0.08 & 0.01 \\
Pelvis start & 0.16 & 0.12 & 0.05 & 0.00 & 0.13 & 0.14 & 0.14 \\
Upper arm start & $\mathbf{- 0 . 5 0}$ & $\mathbf{- 0 . 5 7}$ & $\mathbf{- 0 . 4 7}$ & $\mathbf{- 0 . 4 3}$ & $\mathbf{- 0 . 4 5}$ & $\mathbf{- 0 . 3 9}$ & $\mathbf{- 0 . 4 8}$ \\
Elbow start & $\mathbf{- 0 . 4 2}$ & $\mathbf{- 0 . 3 7}$ & 0.22 & 0.22 & 0.11 & 0.32 & 0.31 \\
Delta head & $\mathbf{- 0 . 3 6}$ & -0.32 & -0.29 & -0.40 & -0.20 & -0.32 & -0.33 \\
Delta trunk & 0.06 & 0.06 & 0.02 & -0.05 & 0.19 & -0.06 & 0.03 \\
Delta pelvis & -0.32 & $\mathbf{- 0 . 3 6}$ & $\mathbf{- 0 . 4 3}$ & -0.28 & -0.01 & -0.30 & $\mathbf{- 0 . 3 8}$ \\
Delta upper arm & -0.09 & -0.21 & -0.11 & -0.07 & $\mathbf{- 0 . 3 5}$ & -0.02 & -0.11 \\
Delta elbow & $\mathbf{- 0 . 5 0}$ & $\mathbf{- 0 . 4 6}$ & $\mathbf{- 0 . 4 3}$ & $\mathbf{- 0 . 4 3}$ & -0.16 & $\mathbf{- 0 . 3 8}$ & $\mathbf{- 0 . 4 8}$ \\
\hline
\end{tabular}

Children with Bi-CP

\begin{tabular}{lrrrrrrr} 
& $\begin{array}{c}\text { PEDI } \\
\text { fssc }\end{array}$ & $\begin{array}{r}\text { PEDI } \\
\text { fsmob }\end{array}$ & $\begin{array}{c}\text { PEDI } \\
\text { fssoc }\end{array}$ & $\begin{array}{c}\text { PEDI } \\
\text { casc }\end{array}$ & $\begin{array}{r}\text { PEDI } \\
\text { camob }\end{array}$ & $\begin{array}{r}\text { PEDI } \\
\text { casoc }\end{array}$ & $\begin{array}{c}\text { PEDI } \\
\text { total score }\end{array}$ \\
\hline Head start & 0.32 & 0.02 & 0.08 & 0.16 & 0.09 & $\mathbf{0 . 5 3}$ & 0.22 \\
Trunk start & -0.09 & 0.25 & -0.18 & -0.26 & 0.19 & -0.17 & 0.04 \\
Pelvis start & -0.05 & 0.14 & -0.10 & -0.16 & 0.30 & 0.17 & 0.08 \\
Upper arm start & 0.10 & -0.11 & -0.09 & 0.00 & -0.19 & -0.10 & -0.00 \\
Elbow start & -0.34 & 0.06 & -0.33 & -0.32 & 0.05 & -0.26 & -0.22 \\
Delta head & 0.03 & -0.10 & 0.41 & 0.22 & 0.08 & $\mathbf{- 0 . 4 8}$ & 0.18 \\
Delta trunk & 0.33 & 0.36 & -0.07 & 0.17 & 0.43 & 0.10 & 0.37 \\
Delta pelvis & -0.22 & 0.04 & $\mathbf{- 0 . 5 1}$ & -0.46 & 0.44 & 0.18 & -0.05 \\
Delta upper arm & 0.13 & 0.17 & 0.14 & 0.07 & 0.19 & -0.10 & 0.16 \\
Delta elbow & -0.16 & -0.16 & -0.17 & -0.17 & 0.02 & -0.29 & -0.11 \\
\hline
\end{tabular}

Values are marked in bold when the relation between the kinematic parameter and the PEDI score was statistically significant $(p<0.05)$. Age-groups are pooled.

"Start" indicates the position of the body part at the onset of reaching; "Delta" points to the displacement of the body-parts during the reaching movement; fssc, functional scale self-care; fsmob, functional scale mobility; fssoc, functional scale social function; casc, caregiver assistance self-care; camob, caregiver assistance mobility; casoc, caregiver assistance social function.

Postural control and kinematics of reaching. In the TD children, initial positions of head, trunk, and pelvis were not related to the kinematics of reaching, but initial position of shoulder and elbow were (Table 3). A more retroflexed position of the upper arm was associated with the presence of less reaching movements consisting of $1 \mathrm{MU}$, and a more flexed elbow was associated with a lower rate of occurrence of reaching movements of $1 \mathrm{MU}$, slower movements and the transport unit covered a relatively smaller proportion of the reaching distance. In the TD children, displacement of body parts during reaching was not related to the kinematics of reaching.

In children with $\mathrm{SH}$, initial position of the head was related to the kinematics of reaching: a more anteflexed head was associated with slower and less straight reaching movements. In contrast to TD children, in children with $\mathrm{SH}$, displacement of body-parts during reaching was related to the kinematics of reaching (Fig. 3; Table 3). In children with SH, a less stable head was associated with slower and less straight reaching movements. In addition, less trunk mobility was associated with worse reaching movements, i.e. reaching movements during which the transport MU covered a relatively smaller part of the reaching distance, longer lasting reaches, and less reaches consisting of one MU (Fig. 3). Furthermore, more elbow displacement was associated with slower reaching movements.

In children with Bi-CP only a few significant relationships between the kinematics of posture and those of reaching were found (Table 3). Unlike in the other groups, in children with $\mathrm{Bi}-\mathrm{CP}$ initial pelvis position was related to reaching: a more anteflexed pelvis was associated with slower reaching movements. Similar to the findings in the children with SH, in the children with Bi-CP, less trunk displacement during reaching was associated with movements during which a smaller part was covered by the transport movement unit (Fig. 3, Table 3).

The ability of TD children and children with CP to modulate EMG amplitude of the dorsal postural muscles with respect to information on initial body configuration was related to the kinematics of reaching. The ability to modulate was associated with the occurrence of reaching movements during which the transport MU covered a relatively larger part of the movement (ability present: $96 \%$ covered by transport MU, ability absent: $\left.89 \% ; \mathrm{F}_{1,5}=18.0, p<0.01\right)$ and with faster reaching movements (ability present: $0.85 \mathrm{~m} / \mathrm{s}$, ability absent: $0.75 \mathrm{~m} / \mathrm{s}$; ANOVA $F_{1,5}=11.3, p<0.01$ ). In addition, the ability to modulate tended to be associated with the straightness of reaching (ability present: 106\%, ability absent: 108\%; ANOVA $\mathrm{F}_{1,5}=3.6, p=0.06$ ) and the duration of reaching (ability present: $1.2 \mathrm{~s}$, ability absent:1.3 s, ANOVA $_{1,5}=3.0$, $p=0.08)$.

\section{DISCUSSION}

The present study demonstrated that initial posture during reaching of children with $\mathrm{CP}$ differed from that of TD children. Body displacement while reaching did not differ between the groups. Yet, it was especially body displacement and less so initial body configuration that was related to the quality of reaching.

Before discussing the pathophysiological and clinical significance of our findings, we would like to address an important methodological point, namely whether the differences in the kinematics of posture between the children with $\mathrm{CP}$ and the TD children should be attributed to the presence of $\mathrm{CP}$ or to preterm birth. There are several findings that suggest that the presence of $\mathrm{CP}$ mainly explained the differences between the groups. First, in the present study and in a previous study 
Table 3. Spearman rank correlations between kinematics of postural control and kinematics of reaching in TD children and children with CP

\begin{tabular}{|c|c|c|c|c|c|c|c|c|c|c|}
\hline & \multicolumn{5}{|c|}{ TD } & \multicolumn{5}{|c|}{$\mathrm{CP}$} \\
\hline & $\begin{array}{c}\text { Reaching } \\
\text { duration }\end{array}$ & $\begin{array}{l}\text { Peak } \\
\text { velo }\end{array}$ & $\begin{array}{l}\text { Index of } \\
\text { curvature }\end{array}$ & $\begin{array}{c}\% \\
\text { trials } \\
1 \mathrm{MU}\end{array}$ & $\begin{array}{c}\% \\
\text { transport } \\
\text { MU }\end{array}$ & $\begin{array}{l}\text { Reaching } \\
\text { duration }\end{array}$ & $\begin{array}{l}\text { Peak } \\
\text { velo }\end{array}$ & $\begin{array}{l}\text { Index of } \\
\text { curvature }\end{array}$ & $\begin{array}{c}\% \\
\text { trials } \\
1 \mathrm{MU}\end{array}$ & $\begin{array}{c}\% \\
\text { transport } \\
\text { MU }\end{array}$ \\
\hline Head start & 0.09 & -0.08 & -0.33 & 0.07 & 0.06 & -0.04 & -0.34 & 0.20 & 0.02 & 0.01 \\
\hline Trunk start & -0.03 & 0.02 & 0.25 & -0.24 & -0.21 & -0.14 & -0.11 & -0.19 & 0.14 & 0.02 \\
\hline Pelvis start & -0.07 & 0.17 & 0.21 & -0.18 & -0.16 & -0.05 & -0.19 & -0.22 & -0.11 & -0.04 \\
\hline $\begin{array}{l}\text { Upper arm } \\
\text { start }\end{array}$ & 0.14 & 0.07 & 0.25 & -0.44 & -0.30 & -0.15 & 0.05 & 0.06 & 0.11 & -0.07 \\
\hline Elbow start & -0.06 & 0.60 & -0.33 & 0.48 & 0.47 & 0.08 & 0.27 & -0.02 & 0.03 & 0.05 \\
\hline Delta head & -0.35 & -0.27 & 0.35 & -0.29 & -0.22 & 0.07 & -0.31 & 0.40 & -0.27 & -0.16 \\
\hline Delta trunk & 0.27 & -0.03 & 0.08 & -0.33 & -0.32 & -0.28 & 0.21 & -0.18 & 0.30 & 0.42 \\
\hline Delta pelvis & -0.11 & -0.02 & 0.11 & -0.28 & -0.29 & -0.27 & 0.04 & 0.13 & 0.11 & 0.11 \\
\hline $\begin{array}{l}\text { Delta upper } \\
\text { arm }\end{array}$ & -0.06 & 0.32 & 0.11 & -0.25 & -0.09 & 0.01 & 0.26 & 0.06 & 0.06 & 0.16 \\
\hline \multirow[t]{3}{*}{ Delta elbow } & 0.11 & -0.17 & 0.13 & -0.31 & -0.29 & 0.02 & -0.26 & 0.16 & -0.07 & -0.15 \\
\hline & \multicolumn{5}{|c|}{ Children with SH } & \multicolumn{5}{|c|}{ Children with Bi-CP } \\
\hline & $\begin{array}{l}\text { Reaching } \\
\text { duration }\end{array}$ & $\begin{array}{l}\text { Peak } \\
\text { velo }\end{array}$ & $\begin{array}{l}\text { Index of } \\
\text { curvature }\end{array}$ & $\begin{array}{c}\% \\
\text { trials } \\
1 \mathrm{MU}\end{array}$ & $\begin{array}{c}\% \\
\text { transport } \\
\text { MU }\end{array}$ & $\begin{array}{c}\text { Reaching } \\
\text { duration }\end{array}$ & $\begin{array}{l}\text { Peak } \\
\text { velo }\end{array}$ & $\begin{array}{l}\text { Index of } \\
\text { curvature }\end{array}$ & $\begin{array}{c}\% \\
\text { trials } \\
1 \mathrm{MU}\end{array}$ & $\begin{array}{c}\% \\
\text { transport } \\
\text { MU }\end{array}$ \\
\hline Head start & 0.07 & -0.41 & 0.35 & -0.09 & -0.15 & -0.16 & -0.23 & -0.08 & 0.23 & 0.26 \\
\hline Trunk start & -0.08 & -0.13 & -0.08 & 0.19 & -0.00 & 0.09 & -0.26 & -0.24 & -0.18 & -0.07 \\
\hline Pelvis start & -0.23 & 0.01 & -0.26 & 0.09 & 0.14 & 0.41 & -0.56 & -0.17 & -0.47 & -0.29 \\
\hline $\begin{array}{l}\text { Upper arm } \\
\text { start }\end{array}$ & -0.02 & 0.04 & -0.02 & 0.04 & -0.15 & -0.39 & 0.15 & 0.04 & 0.35 & 0.25 \\
\hline Elbow start & 0.09 & 0.30 & 0.07 & 0.03 & 0.13 & 0.16 & -0.08 & 0.03 & -0.19 & -0.45 \\
\hline Delta head & 0.08 & -0.35 & 0.57 & -0.27 & -0.30 & 0.18 & -0.24 & 0.11 & -0.25 & 0.10 \\
\hline Delta trunk & -0.41 & 0.33 & -0.13 & 0.40 & 0.48 & -0.12 & 0.13 & -0.35 & 0.40 & 0.62 \\
\hline Delta pelvis & -0.26 & 0.09 & 0.02 & 0.21 & 0.26 & -0.32 & -0.10 & 0.26 & 0.06 & -0.13 \\
\hline $\begin{array}{l}\text { Delta upper } \\
\text { arm }\end{array}$ & 0.06 & 0.16 & 0.08 & 0.02 & 0.14 & 0.01 & 0.40 & 0.02 & 0.13 & 0.15 \\
\hline Delta elbow & 0.20 & -0.37 & 0.22 & -0.12 & -0.34 & -0.32 & -0.03 & 0.10 & 0.13 & 0.33 \\
\hline
\end{tabular}

Values are marked in bold when the relation between the kinematic parameters was statistically significant $(p<0.05)$. Age-groups are pooled.

(paper under review at Pediatric Research) we found that postural control and the quality of reaching were related to the severity of $\mathrm{CP}$, the severity of brain lesion, and to the degree of spasticity, whereas in other studies in young preterm children without CP similar relationships were absent $(8,9,23)$. Second, the finding that the degree of prematurity did not affect the kinematic parameters when the severity of brain lesion was taken into account suggests that the present findings mainly are related to the presence of $\mathrm{CP}$.

Initial sitting position of the children with $\mathrm{CP}$ differed from that of the TD children: children with $\mathrm{CP}$ sat with a more reclined pelvis and a more collapsed trunk. This is a wellknown clinical finding, in particular in children with Bi-CP. It can be attributed partly to an imbalance between the relatively weak hip flexor muscles and overactive hip extensor muscles (24). In addition, it can be explained as the child's strategy to cope with postural instability. For, in the crouched sitting position with pelvic retroflexion, children with Bi-CP are better able to adapt postural muscle activity to environmental conditions than in a sitting position with less pelvic retroflexion (25).

In the TD children the kinematics of posture changed with increasing age. In the first place, head displacement during reaching decreased with increasing age. This finding underscores the notion that head stabilization in space, which is strongly linked to gaze orientation, is one of the major goals of postural control (26). Second, the trunk in the 511 y olds was more flexed than in the younger children. This finding corresponds to the data of Hadders-Algra et al. (27), who reported that trunk flexion especially increases between the ages of 2 and $3 \mathrm{y}$, a phenomenon that is accompanied by a decrease in the in-concert activation of the postural muscles. In the children with CP participating in the present study, the effect of age on postural characteristics was altered in a complex way by the severity of the disability. Children with $\mathrm{CP}$ are able to learn to some extent from previous experience (28), but their motor performance also strongly depends on the severity of their motor disorder.

The present study indicated that the degree of spasticity of the reaching arm as measured by the Modified Ashworth Scale in children with a spastic type of $\mathrm{CP}$ was related to a minor extent only to the kinematic parameters of postural control. Three explanations can be offered for the weak relationships. First, it should be realized that the frequently used Modified Ashworth Scale is only a moderately valid instrument to assess spasticity (29). Second, we studied behavior of the least involved arm in children with CP. It is conceivable that the degree of spasticity of the most involved arm and kinematics of postural control are more closely related than the degree of 

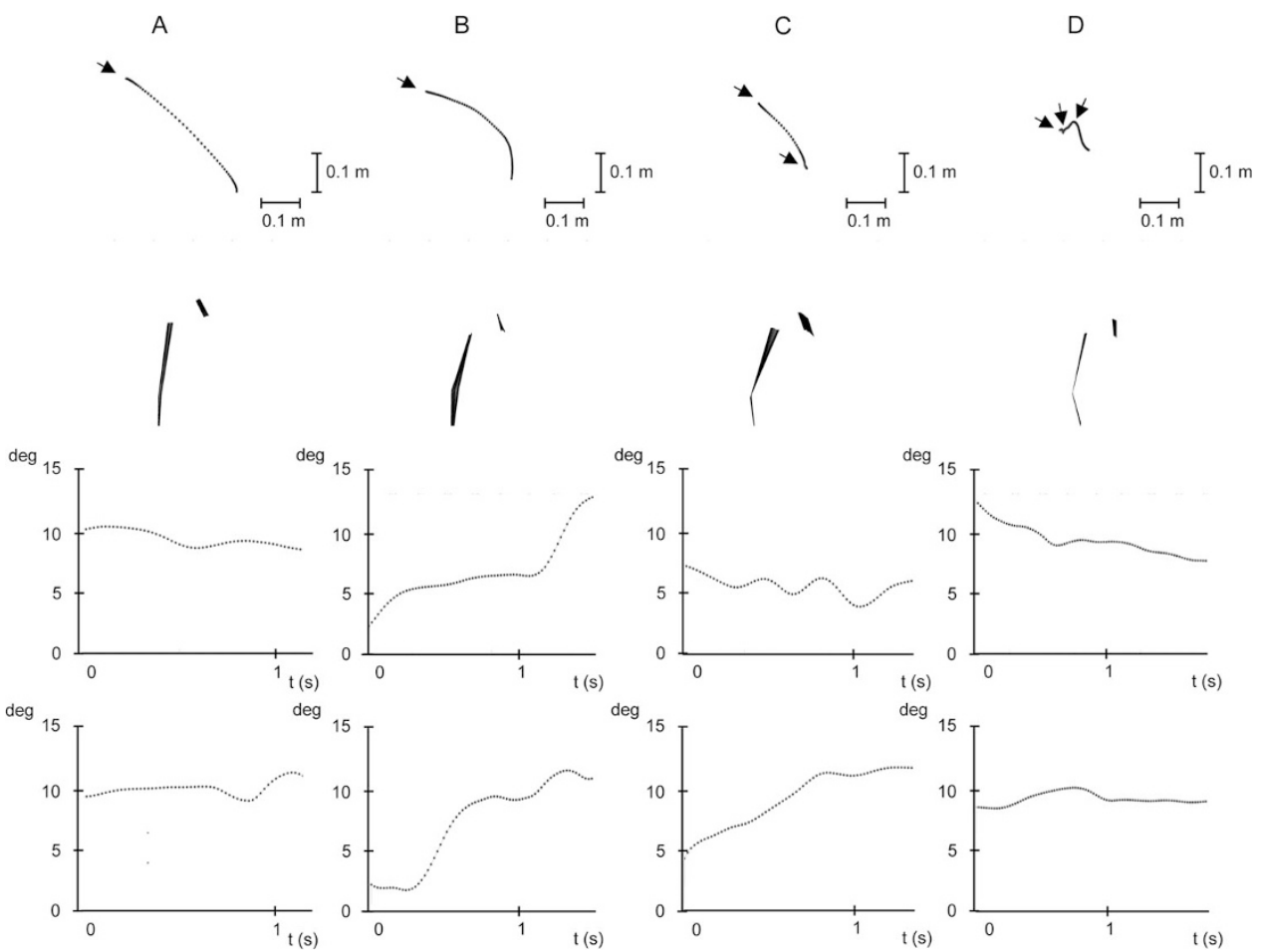

Figure 3. Reaching trajectories, stick diagrams and angular displacement of head and trunk Typical examples of reaching movements of a TD child of $11 \mathrm{y}(A)$, a child with SH of $10 \mathrm{y}(B)$, a child with Bi-CP of $10 \mathrm{y}(C)$ and a child with SH of $7 \mathrm{y}$. The panels show, from top to bottom, for the four children a) a reaching trajectory (first row), b) the stick diagrams of the head and trunk during this reaching movement (second row), c) the angular displacement of the head (third row) and d) the angular displacement of the trunk (bottom row) during this reaching movement. The arrows in the top panels indicate the start of the reaching movement and the beginning and end of a MU. The TD child $(A)$ and the child with SH $(B)$ show a reaching movement of $1 \mathrm{MU}$. The child with Bi-CP $(C)$ and the child with $\mathrm{SH}(D)$ show a reaching movement consisting of $2 \mathrm{MU}$ and $3 \mathrm{MU}$, respectively. Note that the relatively immobile trunk of child $D$ is associated with a reaching movement consisting of $3 \mathrm{MU}$, whereas the more mobile trunks of children $B$ and $C$ are associated with a reaching movement of less MU.

spasticity of the least involved arm and postural kinematics. Third, others have demonstrated that the relationship between spasticity and motor performance in children with $\mathrm{CP}$ is not a straightforward one (30).

The assumption that the quality of reaching depends on the adequacy of postural control is confirmed in the present study, but the interrelationships differed for the three different groups. TD children in general are able to modulate the activity of the postural muscles to task-specific conditions $(13,19)$, a capacity that was related to a better quality of reaching. In the TD children the actual and well-controlled position and displacement of head, trunk, and pelvis were not related to the quality of reaching. Children with SH have a limited capacity to modulate the activity of postural muscles to task-specific conditions (13). In these children, the less-well-controlled posture was related to a worse quality of reaching. Specifically, a more anteflexed starting position of the head, a less stable head, and a less mobile trunk while reaching were related to a worse quality of the reaching movements. The association between a larger displacement of the trunk and better reaching movements suggests that children with SH integrate truncal mobility in the reaching movement by creating a positive link between the two. Children with Bi-CP cannot modulate the activity of the postural muscles to task specific conditions (13). In these children a strong link was also found between little trunk mobility and a worse quality of reaching. In addition, quality of reaching was related to initial pelvis position. The practical implications of these findings could be 3 -fold. First, it is conceivable that the quality of reaching in children with $\mathrm{CP}$ might improve by specific training. For instance, by exercises during which the sitting child reaches to objects placed at such a distance that they require considerable trunk displacement. Second, it is possible that quality of reaching in children with $\mathrm{Bi}-\mathrm{CP}$ benefits from their preferred reclined pelvis position. Third, functional performance of children with CP might profit from the provision of external stabilizers of the pelvis (seating devices) and, in particular in children with a severe form of CP, stabilizing devices for the head.

In conclusion, sitting posture before the onset of reaching of children with $\mathrm{CP}$ differs from that of TD children: they sit with a more reclined pelvis and a more collapsed trunk. This different sitting posture is not related to worse functional performance during daily life activities and the reclined pelvic position even results in a better quality of reaching movements. In addition, a more stable head, a more mobile trunk, and a more stable pelvis were related to better functional performance. This suggests that physiotherapeutic guidance of children with CP should focus rather on the latter postural parameters than on the different sitting posture of pelvis and trunk.

\section{REFERENCES}

1. Gordon J 2000 Assumptions underlying physical therapy intervention: theoretical and historical perspectives. In: Carr J, Shepherd R (eds) Movement Science Foundations for Physical Therapy in Rehabilitation. Gaithersburg, MD, pp 1-31 
2. Lennon S, Baxter D, Ashburn A 2001 Physiotherapy based on the Bobath concept in stroke rehabilitation: a survey within the UK. Disabil Rehabil 23:254-262

3. Kuypers HG. 1981 Anatomy of the descending pathways. In: Brookhart JM, Mountcastle VB (eds) Handbook of Physiology. The Nervous System, Part II. American Physiological Society, Bethesda, MD pp 597-666

4. Massion J 1998 Postural control systems in developmental perspective. Neurosci Biobehav Rev 22:465-472

5. Amiel-Tison C, Grenier A 1983 Neurological Examination of the Newborn and Infant. Masson, New York, pp 1-176

6. Thelen E, Spencer JP 1998 Postural control during reaching in young infants: a dynamic systems approach. Neurosci Biobehav Rev 22:507-514

7. Fallang B, Saugstad OD, Hadders-Algra M 2000 Goal directed reaching and postural control in supine position in healthy infants. Behav Brain Res 115:9-18

8. Fallang B, Saugstad OD, Grøgaard J, Hadders-Algra M 2003 Kinematic quality of reaching movements in preterm infants. Pediatr Res 53:836-842

9. Fallang B, Saugstad OD, Hadders-Algra M 2003 Postural adjustments in preterm infants at 4 and 6 months post term age during voluntary reaching in supine position. Pediatr Res 54:826-833

10. Hopkins B, Rönnqvist L. 2002 Facilitating postural control: effects on the reaching behavior of 6-month-old infants. Dev Psychobiol 40:168-182

11. Von Hofsten C 1979 Development of visually directed reaching: the approach phase. J Hum Mov St 5:160-178

12. von Hofsten C 1991 Structuring of early reaching movements: a longitudinal study. J Mot Behav 23:280-292

13. van der Heide JC, Begeer C, Fock JM, Otten B, Stremmelaar E, Van Eykern LA, Hadders-Algra M 2004 Postural control during reaching in preterm children with cerebral palsy. Dev Med Child Neurol 46:253-266

14. Bohannon RW, Smith MB 1987 Interrater reliability of a modified Ashworth scale of muscle spasticity. Phys Ther 67:206-207

15. Custers JW, Wassenberg-Severijnen JE, Van der Net J, Vermeer A, 't Hart H, Helder PJ 2002 Dutch adaptation and content validity of the "Pediatric Evaluation of Disability Inventory (PEDI)". Disabil Rehabil 24:250-258

16. Palisano R, Rosenbaum P, Walter S, Russel D, Wood E, Galuppi B 1997 Development and reliability of a system to classify gross motor function in children with cerebral palsy. Dev Med Child Neurol 39:214-223

17. Levene MI, Fawer CL, Lamont RF 1982 Risk factors in the development of intraventricular haemorrhage in the preterm neonate. Arch Dis Child 57:410-417
18. De Vries L, Eken P, Dubowitz LM 1992 The spectrum of leukomalacia using cranial ultrasound. Behav Brain Res 49:1-6

19. Van der Heide JC, Otten B, van Eykern LA, Hadders-Algra M 2003 Development of postural adjustments during reaching in sitting children. Exp Brain Res 151:32-45

20. Henderson SE, Sugden DA 1992 The Movement Assessment Battery of Children (MABC).The Psychological Corporation, Kent, pp 1-128

21. Otten E 2000 DataMonster, a software package running on an Apple Macintosh computer for signal analysis and modelling for research in motor control. In: Winters JM, Crago PE (eds) Biomechanics and Neural Control of Movement and Posture.Springer-Verlag, New York, pp 665-667

22. Messier J, Adamovich S, Berkinblit M, Tunik E, Poizner H 2003 Influence of movement speed on accuracy and coordination of reaching movement to memorized targets in three-dimensional space in a deafferented subject. Exp Brain Res 150:399416

23. Van der Fits IB, Flikweert ER, Stremmelaar EF, Martijn A, Hadders-Algra M 1999 Development of postural adjustments during reaching in preterm infants. Pediatr Res 46:1-7

24. Fosang AL, Galea MP, McCoy AT, Reddihough DS, Story I 2003 Measures of muscle and joint performance in the lower limb of children with cerebral palsy. Dey Med Child Neurol 45:664-670

25. Brogren E, Forssberg H, Hadders-Algra M 2001 Influence of two different sitting positions on postural adjustments in children with spastic diplegia. Dev Med Child Neurol 43:534-546

26. Pozzo T, Levik Y, Berthoz A 1995 Head and trunk movements in the frontal plane during complex dynamic equilibrium tasks in humans. Exp Brain Res 106:327-338

27. Hadders-Algra M, Brogren E, Forssberg H 1998 Postural adjustments during sitting at preschool age: presence of a transient toddling phase. Dev Med Child Neurol 40:436-447

28. Gordon AM, Duff SV 1999 Fingertip forces during object manipulation in children with hemiplegic cerebral palsy. I: Anticipatory scaling. Dev Med Child Neurol 41:166-175

29. Pandyan AD, Johnson GR, Price CI, Curless RH, Barnes MP, Rodgers H 1999 A review of the properties and limitations of the Ashworth and modified Ashworth Scales as measures of spasticity. Clin Rehabil 13:373-383

30. Gordon AM, Duff SV 1999 Relation between clinical measures and fine manipulative control in children with hemiplegic cerebral palsy. Dev Med Child Neurol 41:586591 